\title{
Evans blue-mediated white-light detection of non-muscle-invasive bladder cancer: A preclinical feasibility and safety study using a rat bladder urothelial cell carcinoma model
}

\author{
SANNE ELSEN ${ }^{1}$, EVELYNE LERUT ${ }^{2}$, FRANK VAN DER AA ${ }^{3}$, \\ BEN VAN CLEYNENBREUGEL ${ }^{3}$, HENDRIK VAN POPPEL ${ }^{3}$ and PETER DE WITTE ${ }^{1}$ \\ ${ }^{1}$ Laboratory of Molecular Biodiscovery, Faculty of Pharmaceutical Sciences; ${ }^{2}$ Laboratory of Translational Cell and Tissue \\ Research, Faculty of Medicine, KU Leuven; ${ }^{3}$ Department of Urology, University Hospital Leuven, B-3000 Leuven, Belgium
}

Received March 17, 2016; Accepted July 15, 2016

DOI: $10.3892 / \mathrm{mco} .2016 .1043$

\begin{abstract}
Photodynamic diagnosis (PDD) improves the detection of non-muscle-invasive bladder cancer (NMIBC). However, white-light (WL) cystoscopy remains the technique routinely used in urological clinics. A more cost-effective but equally performant alternative to PDD may encompass the use of an intense tumoritropic dye in combination with WL cystoscopy. Using a preclinical setting, we investigated the practical aspects of the use of Evans blue (EB) dye for the possible future detection of NMIBC using WL cystoscopy. A solution of 1 and $5 \mathrm{mM}$ EB was instilled into healthy and AY-27 tumor-bearing rat bladders. The bladders were then rapidly dissected and the inner walls were inspected for EB using WL stereomicroscopy. EB present in the bladders and the plasma was also quantified using high performance liquid chromatography. To assess the effects of repeated instillations on normal rat bladders, EB was instilled for 7 consecutive days, after which time the bladder wall was investigated histologically. To gain insight into the mechanisms underlying the selective accumulation of EB in malignant urothelium, RNA sequencing of urothelial tissue and subsequent comparative analysis were performed, with a specific focus on cell adhesion. The concentrations of EB were substantially higher in malignant bladders compared with those in healthy bladders, matching the blue staining of the inner bladder wall observed by stereomicroscopy. EB was equally present in the plasma of healthy and tumor-bearing subjects, although at low concentrations. Importantly, EB did not cause any abnormalities in the urothelium after 7 days of repeated instillation in normal rats. RNA sequencing of the urothelium indicated an abnormal expression of several genes related to cell adhesion in malignant urothelium compared
\end{abstract}

Correspondence to: Professor Peter De Witte, Laboratory of Molecular Biodiscovery, Faculty of Pharmaceutical Sciences, KU Leuven, O\&N II, Herestraat 49, B-3000 Leuven, Belgium

E-mail: peter.dewitte@pharm.kuleuven.be

Key words: cell adhesion, diagnostic dye, Evans blue, orthotopic rat model, urothelial bladder carcinoma with the normal urothelium. Our findings may be important for future clinical developments in the field of diagnostics for bladder cancer. Implementing the more cost-effective protocol of EB instillations in combination with WL cystoscopy may offer a benefit to patients as well as the healthcare system.

\section{Introduction}

Bladder cancer (BC) is a major health concern worldwide, with an estimated 429,000 new cases leading to 165,000 deaths in 2012 (1). At initial diagnosis, 70-75\% of bladder cancers are classified as non-muscle-invasive urothelial cell carcinomas, i.e., tumors of urothelial origin confined to the mucosa or submucosa (2). The standard treatment for these lesions is endoscopic removal of the malignant tissue, referred to as transurethral resection of the bladder (TURB). A white-light (WL) cystoscope is commonly used during TURB to visualize the tumors. WL cystoscopy is sufficient for the detection of exophytic tumors, but small papillary tumors and flat tumors, such as carcinoma in situ (CIS), are often missed during the procedure, leading to underdiagnosis and, eventually, recurrence and progression (3). To overcome this problem, different compounds have been clinically tested for their capacity to efficiently photodiagnose bladder tumors, including tetracycline, hypericin, Photofrin ${ }^{\circledR}, 5$-aminolevulinic acid and its hexyl ester hexaminolevulinate (HAL, Hexvix ${ }^{\circledR} /$ Cysview $^{\circledR}$ ) (4). HAL-based detection of bladder tumors has been shown to be most efficient and, therefore, is the compound of choice when performing photodynamic diagnosis (PDD) for the detection of bladder tumors (5). Different studies have indicated that PDD following instillation of tumoritropic (pro-) fluorescent compounds improves the detection of tumors in clinical practice $(6,7)$. Despite the proven efficacy of PDD and recommendations by experts when CIS or high-grade tumors are suspected (8), WL cystoscopy remains the technique routinely used in urological clinics. This is mainly due to the costly, specialized equipment that is required to perform PDD.

A possible alternative to PDD may be using a tumoritropic dye with an intense color in combination with WL cystoscopy. Such a compound, Evans blue (EB) dye, was previously investigated in vitro and in vivo. In a study by Roelants et al (9), 
the accumulation of EB was significantly higher in spheroids composed of malignant urothelial cells compared with that in spheroids derived from normal human urothelial cells. Elsen et al (10) investigated the accumulation of EB in an orthotopic rat non-muscle-invasive bladder cancer (NMIBC) model. The biodistribution of EB was examined in the different layers of healthy rat bladders and rat bladders bearing a malignant urothelium by quantifying the fluorescence of EB. It was concluded that EB is selectively taken up by tumor tissue when using a $1 \mathrm{mM}$ instillation concentration, and that this specific accumulation is possibly due to urothelial defects in tumor rat bladders.

In the present study, the feasibility of using EB for the detection of NMIBC in the orthotopic rat model was further investigated. WL stereomicroscopy was applied at a low magnification to visually inspect the accumulation of EB in the malignant and normal inner bladder wall, and the amount of EB present in homogenates of tumor bladders and healthy bladders was quantified. Furthermore, the amount of EB present in the plasma following intravesical instillation was investigated, as were the possible histological adverse effects of repeated EB instillations. The rat NMIBC tumor model was characterized in more detail by a transcriptome analysis of malignant and normal rat urothelium, with a specific focus on cell adhesion. The aim of this analysis was to further investigate our previous hypothesis (10) that EB is able to selectively accumulate in tumor tissue due to defects in the urothelial barrier.

\section{Materials and methods}

Orthotopic AY-27 rat bladder tumor model. A total of 51 female Fisher rats (F-344), aged 10 weeks and weighing 160-200 g, were used in all the experiments (Charles River Laboratories, Lyon, France). All animal procedures were performed in compliance with national and European regulations and were approved by the Animal Care and Ethics Committee of the University of Leuven (approval no. 142/2014). AY-27 cells were used for tumor implantation experiments. Details regarding the catheterization of animals and the tumor inoculation procedure were previously described by our group (10). All the experiments were performed 2 days after the inoculation of AY-27 cells into the rat bladder. At this timepoint, high-grade CIS was present, as confirmed by histological analysis (10).

Accumulation of EB in rat bladders and plasma following intravesical instillation

$E B$ administration and stereomicroscopical inspection of inner wall of rat bladder. Following sedation and catheterization of the animals, the EB solution $(0.3 \mathrm{ml})$ was instilled in the bladders. Two conditions were tested: $1 \mathrm{mM}$ EB instillation over $2 \mathrm{~h}$ and $5 \mathrm{mM}$ EB instillation over $1 \mathrm{~h}$ in healthy rats ( $n=5$ per condition) and rats with bladder tumors $(n=5$ per condition). After instillation, the rat bladders were thoroughly flushed with phosphate-buffered saline (PBS). The rats were then euthanized, the bladders were rapidly dissected and the inner wall was visually inspected for blue dye accumulation using WL stereomicroscopy (Leica MZ10 F; Leica Microsystems GmbH, Wetzlar, Germany). Pictures were captured with a DFC310 FX digital camera. Afterwards, the bladders were extracted (see below).

Blood sampling. Immediately after instillation, the bladders were flushed thoroughly and blood was collected via the tail vein of living animals using a wing needle. The tubes were subsequently centrifuged at $380 \mathrm{x}$ g for $15 \mathrm{~min}$. Three hours after the first blood sampling, blood was collected a second time and processed.

Quantification of EB. The extraction procedure of bladder and blood samples was based on the method described by Gardner (11) using naphtol blue black as an internal standard. The residues of the samples were redissolved in ultrapure water (Milli-Q ${ }^{\circledR}$; Merck Millipore, Darmstadt, Germany) and processed using the LaChrom Elite High Performance Liquid Chromatography (HPLC) system (VWR Hitachi; Hitachi, Tokyo, Japan) equipped with diode array detection (L-2450). Chromatographic separation was performed on a Phenomenex reversed phase column, type Luna $3 \mathrm{u}$ C18 (150x4.6 mm, $3 \mu \mathrm{m})$ attached to a Phenomenex guard column C18 (4x3 mm; Phenomenex, Torrance, CA, USA). The column was operated at a flow rate of $1 \mathrm{ml} / \mathrm{min}$ at $40^{\circ} \mathrm{C}$ (detection wavelength, $614 \mathrm{~nm}$ ). Acetonitrile:phosphate buffer ( $\mathrm{pH} 7,0.067 \mathrm{M}$; 45:55) with $2 \mathrm{mM}$ tetrabutylammonium dihydrogen phosphate was used as the mobile phase.

Statistical analysis. Results are expressed as means \pm standard error of the mean. To compare the concentration of EB under different conditions, a non-parametric Mann-Whitney U test was performed using GraphPad Prism software (GraphPad, La Jolla, CA, USA). The significance level was set at 0.05 .

Repeated intravesical instillation of EB. Healthy rats were instilled with EB solutions for 7 consecutive days during $1 \mathrm{~h}$. Concentrations of 1 and $5 \mathrm{mM}$ were tested, whereas PBS instillations served as control ( $n=3$ per condition). Each day, the rats were anesthetized and catheterized, as previously described. After the last instillation on day 7, the rats were euthanized, the bladders were removed, cut open and immediately transferred into Tissue Tek medium (Miles Inc., Elkart, IN, USA). Consequently, the bladders were snap-frozen in isopentane-cooled liquid nitrogen and cryostat bladder sections were blindly analyzed by a trained histopathologist.

\section{RNA sequencing experiment}

Specimen collection and laser capture microdissection $(L C M)$. Healthy rats $(\mathrm{n}=4)$ and rats bearing a malignant urothelium $(n=6)$ were euthanized, the bladders were dissected, cut open and immediately embedded into Tissue Tek medium (Miles Inc.). The bladders were then snap-frozen in isopentane-cooled liquid nitrogen. Cryostat microtomy was performed to obtain $10-\mu \mathrm{m}$ bladder sections that were mounted onto DNAse- and RNAse-free polyethylene terephthalate membrane-coated metal frame slides $(1.4 \mu \mathrm{m}$, Leica Microsystems $\mathrm{GmbH}$ ). Immediately prior to performing LCM, the cryosections were stained with standard hematoxylin and eosin staining. Urothelial tissue was microdissected using a Leica DM60000B microscope (Leica Microsystems GmbH). The lasered fragments were captured on the cap of $200-\mu 1$ 

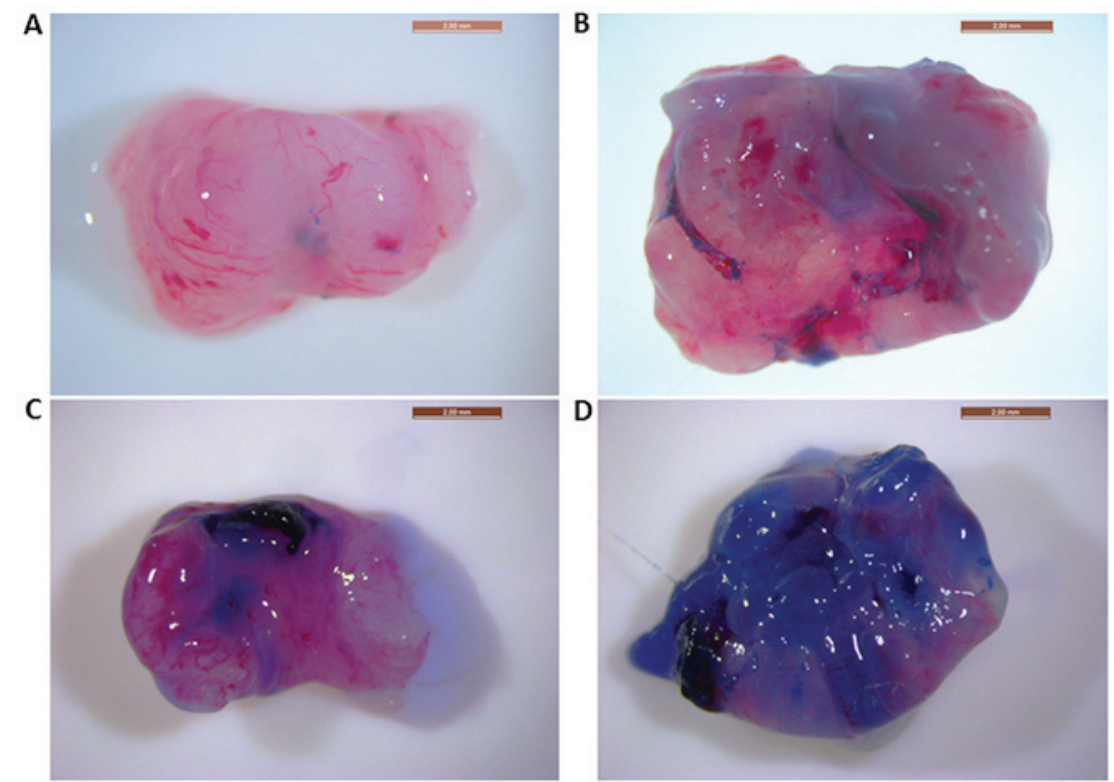

Figure 1. Representative pictures of dissected rat bladders. Pictures of the inner wall of rat bladders after a (A and B) 2-h instillation of $1 \mathrm{mM}$ EB and (C and D) 1-h instillation of $5 \mathrm{mM} \mathrm{EB}$, as visualized by WL stereomicroscopy. Panels A and C show healthy rat bladders, whereas panels B and D show rat bladders bearing a malignant urothelium. Magnification, x1.25. EB, Evans blue dye; WL, white-light.

polymerase chain reaction tubes (Greiner Bio-One, Vilvoorde, Belgium), filled with $25 \mu 1$ RLT plus buffer (Qiagen, Antwerp, Belgium $)+1 \% 2$-mercaptoethanol. For each rat, four cryosections were microdissected.

RNA extraction, sequencing and data analysis. Microdissected tissue from the same rats was pooled together, after which time RNA was extracted according to the protocol described in the RNeasy Plus microkit (Qiagen). Per sample, 1 ng of total RNA was used as input for the SMART-Seq v4 Ultra Low Input RNA protocol (version '040215') from Clontech Laboratories Inc. (Mountain View, CA, USA). Subsequently, $1 \mathrm{ng}$ of purified cDNA was sheared to 300 bp using the Covaris M220 Focused-ultrasonicator (Woburn, MA, USA). Sequencing libraries of each sample were finally equimolarly pooled and sequenced on $1 / 2 \mathrm{NextSeq} 500$ flow-cell at $1 \times 75 \mathrm{bp}$. After cleaning and adapter removal, preprocessed reads were mapped to the Rattus norvegicus reference genome (rnor50) with Tophat v2.0.13 software (https://ccb.jhu.edu/software/tophat/index. shtml). The number of reads in the alignment, overlapping with gene characteristics, were counted using the featureCounts 1.4.6 program (http://subread.sourceforge.net/) (12). Subsequently, genes with less than one count-per-million were removed and normalization was performed for GC content, library size and RNA composition using the EDASeq package from Bioconductor (Copenhagen, Denmark) (13). Principal component analysis was conducted to identify expression patterns in the dataset. To obtain the differentially expressed genes between the two conditions, statistical comparative analysis was performed with EdgeR 3.4.0 (Bioconductor) (14). The resulting P-values were corrected for multiple testing with Benjamini-Hochberg to control the false discovery rate (FDR) (15). Results are presented as $\log _{2}$ fold change and a FDR P-value $<0.05$ was considered to indicate statistically significant differences. Genes with a $\log _{2}$-ratio $<-1$ and $>1$ were considered as down- and upregulated, respectively.
Functional enrichment analysis. In order to convert the dataset into more biologically interpretable data, a functional enrichment analysis was performed. Since pathways in rats are poorly annotated and the majority of databases are made for human genes, the putative orthologous genes in human were extracted for the 14,115 genes annotated in the rat genome used for the statistical analysis. A total of 10,740 orthologous genes were obtained using one-to-one orthologous relationship from Ensembl (BioMart Portal v0.9; http://www.ensembl. org/index.html). The gene enrichment analysis was performed online with WebGestalt (WEB-based Gene SeT AnaLysis Toolkit; http://bioinfo.vanderbilt.edu/webgestalt/) using our sets of differentially expressed genes. The pathway analysis was performed using the annotated pathways of the Kyoto Encyclopedia of Genes and Genomes (KEGG) database, with a default significance level of 0.05 (FDR-corrected P-values).

\section{Results}

Accumulation of EB in rat bladders and plasma following intravesical instillation

Visual inspection of inner wall of rat bladder. Representative images of the inner wall of rat bladders following instillations of $1 \mathrm{mM} \mathrm{EB}$ for $2 \mathrm{~h}$ and $5 \mathrm{mM}$ EB for $1 \mathrm{~h}$, as visualized by WL stereomicroscopy, are shown in Fig. 1. In healthy bladders instilled with the lowest concentration of $\mathrm{EB}$, the blue staining is almost non-existent (Fig. 1A), while a clear blue, non-homogeneous staining is visible in the tumor bladders (Fig. 1B). Healthy bladders instilled with the highest concentration exhibited some non-specific blue staining (Fig. 1C), whereas tumor bladders exhibited an intense blue color (Fig. 1D). Under both conditions, the healthy rat bladders were less stained when compared with those bearing tumors.

In order to compare the amount of EB present in healthy and malignant rat bladders following intravesical instillation, EB was extracted and quantified using an HPLC method. 
Determination of $E B$ in rat bladder homogenates following intravesical instillation. A quantitative comparison of the concentration of EB in bladder homogenates following instillation of the compound in healthy rat bladders and in bladders with malignant urothelium is shown in Fig. 2: Graph 2 indicates that under both tested conditions, more EB was taken up by tumor-bearing compared with healthy bladders, although this difference was only found to be significant in the 1 $\mathrm{mM}-2 \mathrm{~h}$ condition. On average, the concentration of EB in tumor bladder homogenates was 5.5 times higher compared with that in healthy bladder homogenates in the $1 \mathrm{mM}-2 \mathrm{~h}$ condition. In the $5 \mathrm{mM}-1 \mathrm{~h}$ condition, a 3.2-fold increase was observed. These findings match the observations of blue color in the bladder samples, as visualized in Fig. 1.

Determination of EB in rat plasma following intravesical instillation. To determine the amount of EB that evades from the bladder and enters the circulation, the concentration of EB in rat plasma was quantified following intravesical instillation in both healthy and malignant rat bladders. EB concentrations were also determined $3 \mathrm{~h}$ after removal of the catheters to investigate retention of EB in rat blood. As shown in Fig. 3, there were no significant differences in EB concentrations between the plasma of normal rats and the plasma of rats with a malignant urothelium following instillation of EB under all tested conditions ( $1 \mathrm{mM}$ for 2 and $5 \mathrm{~h}$, and $5 \mathrm{mM}$ for 1 and $4 \mathrm{~h}$ ). As expected, the 5-mM instillation led to a higher concentration of $\mathrm{EB}$ in the plasma compared with the 1-mM instillation (3- to 4-fold increase). The highest mean concentration in rat plasma, $7 \mu \mathrm{g} / \mathrm{ml}$, was observed for the $5 \mathrm{mM}$ for $1 \mathrm{~h}$ condition in healthy rat bladders. After a 3-h EB instillation-free period, the EB concentrations did not significantly change.

Repeated intrabladder instillation of EB. EB solutions or PBS (control) were instilled for 7 consecutive days in normal rat bladders to investigate possible adverse effects of EB on the bladder wall. In the control PBS samples (Fig. 4C), the urothelium appeared normal in all 3 rat bladders; in 2 bladders, a purulent inflammation was found in the muscularis, while in the third rat, oedema was present in the lamina propria. The histological injuries seen under these control conditions were likely due to the repeated instillation procedure.

A 7-day repeated instillation of $1 \mathrm{mMEB}$ was not associated with any more signs of adverse effects observed within the bladder wall as compared with the control conditions (Fig. 4A). Histological analysis revealed that the urothelium was normal in all cases. Mild inflammation and oedema was noticed in the lamina propria and 1 bladder had a purulent inflammation in the muscularis. In addition, in cases of $5 \mathrm{mM}$ EB instillations, the urothelium of the 3 rat bladders was normal (Fig. 4B), while inflammation and oedema were observed in the lamina propria and the muscularis of 2 rats.

RNA sequencing experiment. In order to better understand the differential accumulation of EB in urothelial tumor tissue vs. healthy tissue, an RNA sequencing experiment was designed to specifically investigate the gene expression of urothelial tissue. To isolate the urothelium, laser capture microdissection was performed.
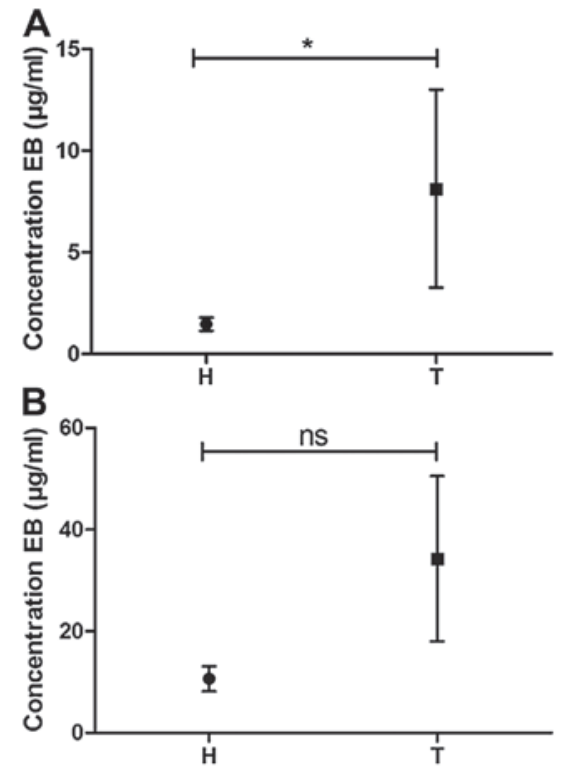

Figure 2. Concentration of EB in bladder homogenates following intravesical instillation. The concentration of EB found in homogenates of healthy bladders $(\mathrm{H})$ and bladders bearing tumors $(\mathrm{T})$ following intravesical instillation is compared. Two conditions were tested: (A) $1 \mathrm{mM}$ instillation of EB for $2 \mathrm{~h}$ and (B) $5 \mathrm{mM}$ instillation of $\mathrm{EB}$ for $1 \mathrm{~h}$ ( $\mathrm{n}=5$ for each condition). Means \pm standard error of the mean are shown. "P<0.05. EB, Evans blue dye; ns, non-significant.

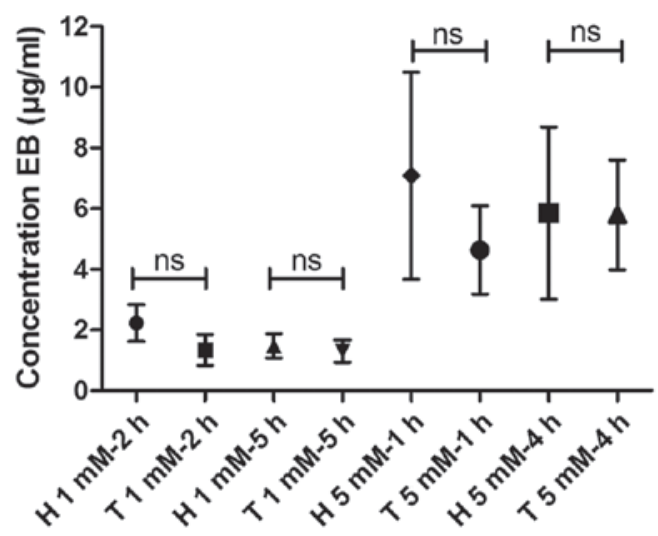

Figure 3. Concentration of EB in bladder plasma following intravesical instillation. The concentration of EB found in rat plasma following intravesical instillation was compared between healthy bladders $(\mathrm{H})$ and bladders bearing tumors $(\mathrm{T})$. Four conditions were tested: $1 \mathrm{mM}$ EB instillation for $2 \mathrm{~h}$ $(1 \mathrm{mM}-2 \mathrm{~h}), 5 \mathrm{mM}$ EB instillation for $1 \mathrm{~h}(5 \mathrm{mM}-1 \mathrm{~h})$ and the blood samples collected $3 \mathrm{~h}$ after the end of instillation ( $1 \mathrm{mM}-5 \mathrm{~h}$ and $5 \mathrm{mM}-4 \mathrm{~h})(\mathrm{n}=3$ for each condition). Means \pm standard error of the mean are shown. EB, Evans blue dye; ns, non-significant.

Comparative analysis between tumor and healthy samples. Principal component analysis demonstrated that 1 tumor sample did not cluster well with the other 5 tumor samples. This sample was therefore considered as an outlier and was excluded from the comparative analysis. Comparative analysis of the remaining tumor samples and the 4 healthy samples revealed that 2,441 genes were downregulated $\left(\log _{2}\right.$-ratio <-1) and 2,244 genes were upregulated $\left(\log _{2}\right.$-ratio $\left.>1\right)$ in tumor vs. healthy samples $(\mathrm{P}<0.05)$, as visualized by a Volcano plot (Fig. 5).

Since we previously hypothesized that EB specifically accumulates in tumor tissue due to defects in the urothelial 
Table I. Differential expression of cell adhesion genes in tumor vs. healthy samples.

\begin{tabular}{|c|c|c|c|c|c|c|c|c|}
\hline \multicolumn{3}{|c|}{ Tight junctions } & \multicolumn{3}{|c|}{ Adherens junctions } & \multicolumn{3}{|c|}{ Desmosomes } \\
\hline Gene ID & LogFC & P-value & Gene ID & $\operatorname{LogFC}$ & P-value & Gene ID & LogFC & P-value \\
\hline Amotll & -4.45 & $1.86 \mathrm{E}-07$ & Acpl & 1.11 & 0.00527 & $D s c 2$ & 1.99 & 4.13E-06 \\
\hline Cldn3 & 7.89 & $9.90 \mathrm{E}-39$ & $A c t b$ & 3.00 & $3.51 \mathrm{E}-07$ & $D s p$ & -1.64 & 0.00120 \\
\hline Cldn 8 & -6.78 & $3.00 \mathrm{E}-25$ & Actgl & 2.55 & 0.00552 & Pkpl & 1.16 & 0.00179 \\
\hline Cldn11 & -2.90 & 0.00956 & Actnl & 3.18 & $3.05 \mathrm{E}-07$ & & & \\
\hline Cldn18 & 3.85 & 0.00786 & Actn4 & 1.32 & $1.42 \mathrm{E}-06$ & & & \\
\hline Cldn23 & -5.07 & $2.14 \mathrm{E}-13$ & Baiap2 & 1.51 & $6.38 \mathrm{E}-06$ & & & \\
\hline F11r & -1.22 & $7.62 \mathrm{E}-05$ & $C d h 3$ & 2.01 & 0.00576 & & & \\
\hline Inadl & -1.51 & 0.0238 & $C d h 4$ & -3.42 & 0.0187 & & & \\
\hline Jam2 & -2.99 & 0.00219 & $C d h 17$ & 4.18 & 0.00247 & & & \\
\hline Jam3 & -2.35 & 0.0400 & Cdh18 & -2.97 & 0.0168 & & & \\
\hline Magi3 & -1.33 & 0.00173 & Csnk2b & -1.39 & $1.21 \mathrm{E}-07$ & & & \\
\hline$M p d z$ & 2.74 & 0.00940 & Ctnndl & -1.26 & 0.00193 & & & \\
\hline Pard6a & 4.02 & $4.76 \mathrm{E}-05$ & Insr & -1.90 & 0.0150 & & & \\
\hline Pard6b & 1.63 & $2.03 \mathrm{E}-05$ & Ptpnl & 1.92 & $1.19 \mathrm{E}-06$ & & & \\
\hline Pard6g & -4.48 & $1.13 \mathrm{E}-13$ & Ptprb & -3.54 & 0.00075 & & & \\
\hline Prkca & 1.92 & 0.00302 & Ptprf & -1.41 & $4.90 \mathrm{E}-06$ & & & \\
\hline Prkch & -1.97 & 0.00018 & $P V R$ & 2.11 & $4.36 \mathrm{E}-11$ & & & \\
\hline Prkci & -1.26 & 0.00011 & Pvrll & -3.13 & 0.00099 & & & \\
\hline Prkcq & -6.70 & $5.77 \mathrm{E}-13$ & Pvrl3 & -3.68 & 0.00333 & & & \\
\hline$R a b 3 b$ & 4.33 & 0.00065 & Pvrl4 & -2.43 & 9.39E-07 & & & \\
\hline \multirow[t]{3}{*}{$Y b x 3$} & 1.63 & $1.18 \mathrm{E}-06$ & Snai2 & -2.76 & 0.00619 & & & \\
\hline & & & Was & 4.01 & 0.00055 & & & \\
\hline & & & Wasl & -1.17 & $9.02 \mathrm{E}-05$ & & & \\
\hline
\end{tabular}

Green, downregulated genes; red, upregulated genes. Corrected P-values were used. LogFC, $\log _{2}$-ratio, as calculated by EdgeR.
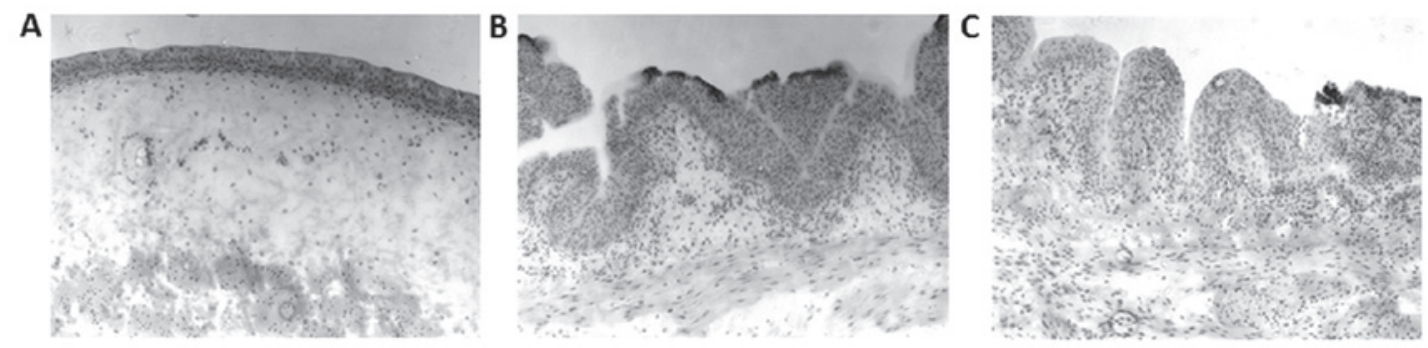

Figure 4. Representative hematoxylin and eosin (H\&E) stainings of 5- $\mu \mathrm{m}$ sections of rat bladders. Photomicrographs of the H\&E-stained sections after a daily 1-h instillation of EB or PBS for 7 consecutive days. (A) $1 \mathrm{mM} \mathrm{EB,} \mathrm{(B)} 5 \mathrm{mM} \mathrm{EB}$, (C) PBS; magnification, x100. EB, Evans blue dye; PBS, phosphate-buffered saline.

barrier (11), genes correlated to cell-cell adhesion were selected and the expression levels were analyzed. The focus was set on genes belonging to or correlated with the three typical cell adhesion structures: Tight junctions (TJs), adherens junctions (AJs) and desmosomes. The results are presented in Table I. This table clearly shows that several genes related to cell adhesion were differentially expressed in the malignant urothelium compared with normal urothelium. When investigating genes related to TJs, 13 genes were found to be downregulated, whereas 8 genes were upregulated. These genes include five different types of claudins, the major building blocks of TJs. It is also clear that a number of these genes were up- or downregulated at a very high level, e.g., $C L D N 3$ was 237 -fold upregulated, whereas CLDN23 was 110 -fold downregulated in the tumor urothelium vs. normal urothelium. In addition, genes encoding proteins involved in AJs were differentially expressed, although at a lower level compared with the TJ-related genes. Highest levels of upregulation were observed for $C D H 17$ (18-fold) and of downregulation for PVRL3 (13-fold). Furthermore, 3 genes correlated with desmosomes were detected, namely $D S C 2$, $D S P$ and $P K P 1$. 
Functional enrichment analysis. To identify enriched pathways (PWs), our gene set was compared to predefined categories of the KEGG database. This analysis revealed that the upregulated differentially expressed genes were associated with 99 KEGG pathways (Table II). The top 10 of these upregulated enriched PWs is indicated in red. Notably, this table demonstrates that the most significantly enriched PW is the cell cycle PW. In relation to this PW, the DNA replication and mitogen-activated protein kinase signaling PWs are also included in the top 10. Alongside this top 10, other interesting categories that were significantly enriched are the bladder cancer $\mathrm{PW}(\mathrm{R}=5.21, \mathrm{P}=0.0054)$, the $\mathrm{TJ} \mathrm{PW}(\mathrm{R}=3.34$, $\mathrm{P}=0.0025)$ and the $\mathrm{AJ} \mathrm{PW}(\mathrm{R}=3.62, \mathrm{P}=0.012)$.

When analyzing the downregulated differentially expressed genes, 53 different PWs were enriched (Table II). The top 10 is marked in red. Interestingly, several of the PWs in this top 10 are associated with catabolism, in which the cells break down molecules into smaller units to release energy: Lysosome PW, peroxisome $\mathrm{PW}$ and endocytosis $\mathrm{PW}$ are among this category. The peroxisome proliferator-activated receptor signaling, terpenoid backbone synthesis, fatty acid metabolism and soluble NSF attachment protein receptor interactions in vesicular transport PWs, are also indirectly involved in the catabolism of the cells. Other interesting categories that were significantly enriched are the TJ PW $(\mathrm{R}=3.36, \mathrm{P}=0.0055)$ and the $\mathrm{p} 53$ signaling $\mathrm{PW}(\mathrm{R}=3.92, \mathrm{P}=0.017)$.

\section{Discussion}

In this study, using a preclinical setting, we investigated certain practical aspects of the future use of EB as a diagnostic dye in the detection of NMIBC in combination with WL cystoscopy. Furthermore, by performing transcriptome analysis of malignant and normal rat urothelium, we set out to mechanistically elucidate the accumulation of the compound in malignant bladder urothelium.

Our results demonstrated that the total amount of EB accumulating in rat bladders bearing a malignant urothelium was clearly higher compared with that in healthy bladders, at least in case of the 1-mM EB instillation. These data are in line with a previous study using an identical tumor model that quantified the fluorescence of EB at the microscopic level in the different layers of the bladder wall (10). Consequently, a differential uptake of EB by the malignant and normal inner bladder wall was also observed using WL stereomicroscopy. The previous study (10) also demonstrated that EB readily penetrates through the malignant urothelium, reaching and afterwards accumulating in the deeper muscle layers and, hence, the perpendicular stereomicoscopic view at the surface of the inner wall (as in case of a cystoscope) likely visualizes both. The images furthermore revealed a non-homogeneous distribution of the blue color, which probably reflects the variable proliferation of the AY-27 cells after inoculation, as previously reported (10).

Although the differences in the accumulation of EB in bladders with tumor vs. healthy bladders were clear, this was not the case for the EB plasma levels. We expected to find a lower concentration of EB in the plasma of healthy rats due to the presence of a functional urothelial barrier, as compared with the disrupted barrier in the rats bearing bladder tumors.

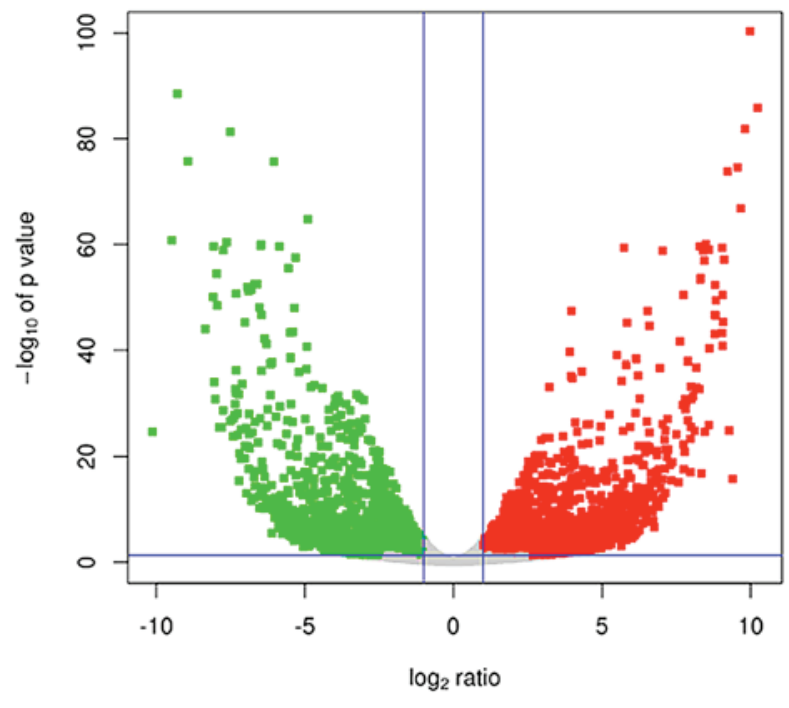

Figure 5. Volcano plot for tumor vs. healthy samples. The vertical lines correspond to a $\log _{2}$-fold change of -1 and 1 , while the horizontal line indicates a P-value of 0.05 . The dots are colored green or red if classified as down- or upregulated, respectively.

However, it is possible that the catheterization procedure, also under control conditions, caused small injuries, which enabled EB to penetrate into the bloodstream without the need to pass through the urothelial barrier. This possibility should be further investigated using larger animals with bladders that are easily accessible.

Approximately 5\% of the amount of EB instilled was able to enter the bloodstream under the tested conditions. Although this quantity may be an overestimation due to the specific instillation conditions used, the absorption across the bladder wall of EB into the bloodstream may raise some safety concerns. At this point, it should be mentioned that EB has been frequently used as a dye marker to determine blood volume in humans (16-19). To that end, EB is injected directly into the bloodstream. Based on an average blood volume of 51 for an individual weighing $70 \mathrm{~kg}(20)$, the final concentration of EB in the circulatory system in humans ranges between 6.36 (19) and $12.73 \mu \mathrm{g} \mathrm{EB} / \mathrm{ml}$ plasma (17) following intravenous injections. These values are in the range of the highest mean concentration observed in the present study $(7 \mu \mathrm{g} / \mathrm{ml})$. Moreover, Gibson and Gregerson (21) concluded that doses of $\mathrm{EB} \leq 20 \mathrm{mg} / \mathrm{kg}$ are non-toxic when administered intravenously to growing rats. For an individual weighing $70 \mathrm{~kg}$, this dose would result in a plasma concentration of $\sim 500 \mu \mathrm{g} \mathrm{EB} / \mathrm{ml}$ plasma, which is 70-fold higher compared with the highest mean concentration observed in our study. Other uses of EB in clinical practice include the detection of aspirated materials in patients with tracheostomy (22) and imaging in cardioscopy (23). Overall, the widespread use of EB for different applications in clinical practice appears to indicate that, even when intravesically instilled EB leaks into the bloodstream, the safety risk for the patients is deemed very low.

Of note, no adverse effects on the urothelium were observed after daily instillation of EB for 7 days, even after intravesical application of concentrations as high as $5 \mathrm{mM}$. Since the urothelium is directly exposed to the EB solutions, these data again underline the fact that the compound possesses a highly 
Table II. Enriched categories in up- and downregulated genes using the Kyoto Encyclopedia of Genes and Genomes (KEGG) database.

Enriched KEGG PWs for the upregulated genes

in order of significance
Enriched KEGG PWs for the downregulated genes in order of significance

\section{Cell cycle}

Metabolic pathways

Smallcell lung cancer

DNA replication

Pathways in cancer

Focal adhesion

Oocyte meiosis

MAPK signaling pathway

Regulation of actin cytoskeleton

RNA transport

Osteoclast differentiation

Leukocyte transendothelial migration

Chemokine signaling pathway

Spliceosome

Cytokine-cytokine receptor interaction

Leishmaniasis

Progesterone-mediated oocyte maturation

p53 signaling pathway

Pyrimidine metabolism

NOD-like receptor signaling pathway

Chronic myeloid leukemia

Purine metabolism

Protein processing in endoplasmic reticulum

Glioma

Homologous recombination

Pancreatic cancer

Apoptosis

Hematopoietic cell lineage

Amoebiasis

Prostate cancer

Neurotrophin signaling pathway

Jak-STAT signaling pathway

Rheumatoid arthritis

Tcell receptor signaling pathway

Nucleotide excision repair

Shigellosis

Ribosome biogenesis in eukaryotes

Mismatch repair

ECM-receptor interaction

Base excision repair

Phagosome

Toxoplasmosis

Arginine and proline metabolism

Adipocytokine signaling pathway

Non-smallcell lung cancer

Fc gamma R-mediated phagocytosis

Pathogenic Escherichia coli infection

Galactose metabolism

Fc epsilon RI signaling pathway

Toll-like receptor signaling pathway

Colorectal cancer
Metabolic pathways

Lysosome

Valine, leucine \& isoleucine degradation

Peroxisome

PPAR signaling

Endocytosis

Fatty acid metabolism

Terpenoid backbone synthesis

SNARE interactions in vesicular transport

Glutathione metabolism

Propanoate metabolism

Biosynthesis of unsaturated fatty acids

Protein processing in endoplasmic reticulum

Drug metabolism-cytochrome P450

Metabolism of xenobiotics by cytochrome P450

Tryptophan metabolism

Sphingolipid metabolism

Steroid hormone biosynthesis

TGF- $\beta$ signaling pathway

Fatty acid elongation in mitochondria

Steroid biosynthesis

Tight junction

Cysteine and methionine metabolism

Insulin signaling pathway

Circadian rhythm-mammal

Basal cell carcinoma

Hedgehog signaling pathway

Inositol phosphate metabolism

Phosphatidylinositol signaling system

Melanogenesis

Aldosterone-regulated sodium reabsorption

Collecting duct acid secretion

Pathways in cancer

p53 signaling pathway

Amino sugar and nucleotide sugar metabolism

Epithelial cell signaling in Helicobacter pylori infection

Adipocytokine signaling pathway

Fc gamma R-mediated phagocytosis

Other glycan degradation

Glyoxylate and dicarboxylate metabolism

Calcium signaling pathway

Pancreatic secretion

Regulation of actin cytoskeleton

Amoebiasis

Pyruvate metabolism

Retinol metabolism

Proximal tubule bicarbonate reclamation

Glycolysis/gluconeogenesis

Salivary secretion

Lysine degradation

Vascular smooth muscle contraction 
Table II. Continued.

Enriched KEGG PWs for the upregulated genes

in order of significance

\section{Proteasome}

Chagas' disease (American trypanosomiasis)

Hypertrophic cardiomyopathy

Glycolysis/gluconeogenesis

Tight junction

Glutathione metabolism

Dilated cardiomyopathy

Malaria

Ubiquitinmediated proteolysis

Bacterial invasion of epithelial cells

Melanoma

Insulin signaling pathway

Vibrio cholerae infection

Arrhythmogenic right ventricular cardiomyopathy

B-cell receptor signaling pathway

Cytosolic DNA-sensing pathway

VEGF signaling pathway

Arachidonic acid metabolism

Glycerophospholipid metabolism

Endocytosis

Bladder cancer

Glycosphingolipid biosynthesis-lacto and neolacto series

TGF- $\beta$ signaling pathway

Natural killer cellmediated cytotoxicity

Glycosphingolipid biosynthesis-ganglio series

Complement and coagulation cascades

Viral myocarditis

RNA degradation

Glycerolipid metabolism

RIG-I-like receptor signaling pathway

Adherens junction

Amyotrophic lateral sclerosis

Staphylococcus aureus infection

Acute myeloid leukemia

Hepatitis C

Nitrogen metabolism

ErbB signaling pathway

Protein export

Type I diabetes mellitus

Epithelial cell signaling in Helicobacter pylori infection

Renal cell carcinoma

Folate biosynthesis

RNA polymerase

Histidine metabolism

Axon guidance

Drug metabolism-other enzymes

Endometrial cancer

mTOR signaling pathway
Enriched KEGG PWs for the downregulated genes in order of significance

Synthesis and degradation of ketone bodies

Vasopressin-regulated water reabsorption

Red, top 10 of the upregulated enriched PWs; green, top 10 of the downregulated enriched PWs.PWs, pathways; MAPK, mitogen-activated protein kinase; NOD, nucleotide-binding oligomerization domain; mTOR, mammalian target of rapamycin; Jak-STAT, Janus kinase-signal transducer and activator of transcription; ECM, extracellular matrix; VEGF, vascular endothelial growth factor; RIG-I, retinoic acid-inducible gene 1; PPAR, peroxisome proliferator-activated receptor; SNARE, soluble NSF attachment protein receptor; TGF, transforming growth factor. 


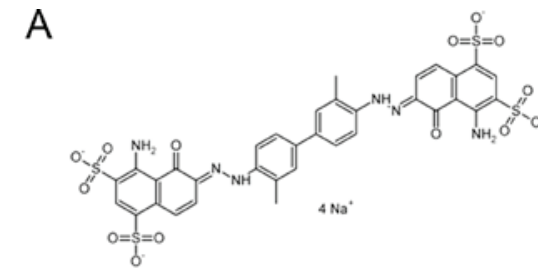

Paracellular route

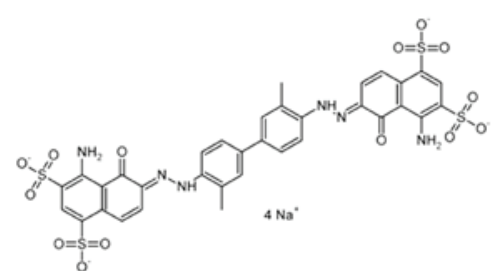

Transcellular route
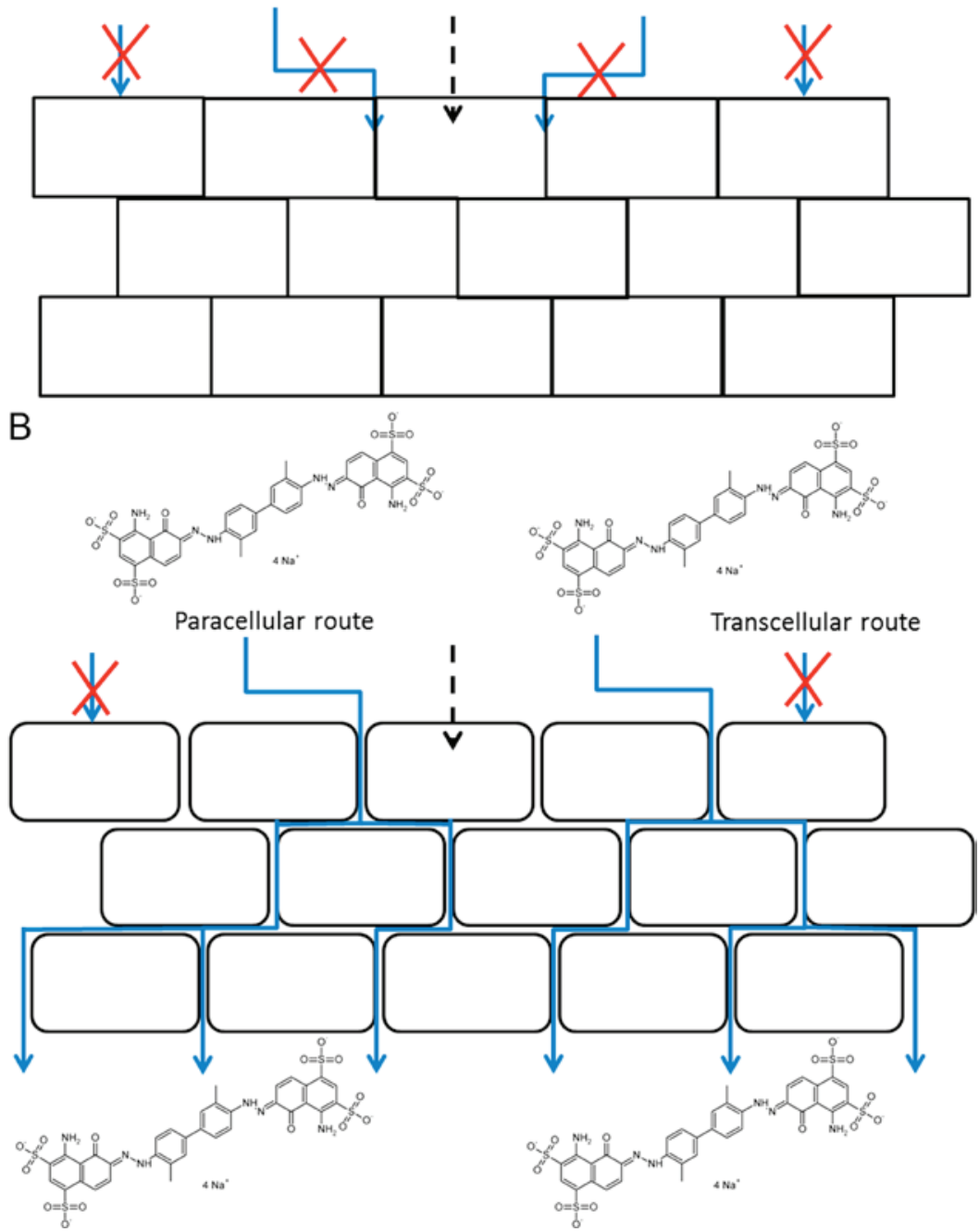

Figure 6. 'Defective barrier' hypothesis. (A) Normal urothelial cells are tightly joined and EB molecules cannot cross this barrier. (B) Tumor cells are characterized by defective cell adhesion structures, thereby enabling EB molecules to pass via the paracellular route. EB, Evans blue dye.

non-toxic profile. However, some inflammation was noticed in the other layers, namely the lamina propria and muscularis, of some, but not all, rat bladder samples. Since these injuries were also present in the control bladders, we hypothesize that the inflammation observed was due to the repeated instillation procedure and not to adverse effects induced by EB per se.

We previously hypothesized that EB selectively accumulates paracellularly in tumor tissue due to defects in the urothelial barrier (10). To investigate this hypothesis in more detail, transcriptome analysis of the urothelium was performed and the differential expression between healthy and malignant urothelium was analyzed. It is clear from the data that several genes related to cell adhesion were differentially expressed, particularly genes associated with TJs. Furthermore, genes that are associated with AJs and desmosomes were found to be significantly up- or downregulated.
A first important observation is the presence of 5 claudin genes, differentially expressed with high values. Claudin proteins are the most important components of TJs. A study investigating the expression of claudins in normal and neoplastic human tissues concluded that most claudin genes are decreased in tumors, except CLDN3, CLDN4 and CLDN7, which are elevated in certain types of cancer, including bladder cancer (24). This conclusion matches our results, in which CLDN8, 11 and 23 were significantly downregulated in malignant urothelium, while $C L D N 3$ was 237 times upregulated.

When investigating in more detail the differentially expressed genes correlated with AJs, four differentcadherins and one catenin, the major building blocks of AJs, were identified. The most extensively investigated cadherin is E-cadherin (CDH1), the most important mediator of cell-cell adhesion in epithelial tissues, and also an important tumor suppressor (25). 
It is well documented that, during epithelial-to-mesenchymal transition (EMT), an important first step of cancer metastasis, a cadherin switch occurs. In different malignancies, it was observed that the expression of E-cadherin decreases, while the expression of $\mathrm{N}$-cadherin $(\mathrm{CDH} 2)$ and/or P-cadherin (CDH3) increases (26). In bladder cancer, a transition to $\mathrm{N}$-cadherin and P-cadherin has also been observed, although the exact timing and combination of expression of the three cadherins remains to be elucidated (26). When these studies are considered in the light of our context, we may see that $\mathrm{P}$-cadherin is indeed significantly upregulated four times in tumor tissue compared with healthy tissue, but the expression of E-cadherin and N-cadherin was not significantly different. This observation may be explained in two ways: It is possible that, in our tumor model, the cadherin switch starts with an increased expression of P-cadherin and that a decreased E-cadherin expression starts later during tumorigenesis. Another possibility is that the EMT has not yet started, and that the P-cadherin expression should be considered separately. Bryan et al (27) investigated the expression of P-cadherin in vivo and concluded that, even in the presence of normal E-cadherin expression, an increased expression of P-cadherin was associated with a more malignant phenotype of bladder cancer. The protein expression of E-cadherin in an identical animal model was previously investigated by our group by means of immunohistochemistry (10). It was concluded that no differences in the expression of E-cadherin were observed, confirming the gene expression of the $C D H 1$ gene in this study. Two other proteins were investigated by Elsen et al (10): Claudin-1 and desmoglein-1. The CLDI and DSG1 genes were not differentially expressed in our gene analysis, but we observed decreased expression of desmoglein-1 in the malignant urothelium in our previous study (10).

Interestingly, the enrichment analysis points to the involvement of cell adhesion as well: The TJ PW was enriched in the upregulated as well as in the downregulated genes, whereas the AJ PW was enriched in the upregulated genes.

Taken together, the data show that several genes involved in cell adhesion are differentially expressed in healthy and malignant urothelium, pointing strongly to defects in the urothelial barrier. Although the relative expression of genes does not necessarily reflect the abundance of the corresponding proteins, it is anticipated that major defects are also present at the level of protein expression. Indeed, our results are fully in line with the previously observed ultrastructural defects, as malignant tissue displayed wider intercellular spaces and a decreased number of cell junction components compared with normal tissue (10).

In conclusion, we demonstrated that EB is selectively taken up by tumor tissue following intravesical instillations in rats bearing bladder tumors compared with normal rats. EB instillations do not harm the bladder wall and penetration into the circulatory system is limited, particularly when low concentrations are used. The selective uptake of EB is probably due to defects in the urothelial barrier, as depicted in Fig. 6.

If these preclinical results on EB can be confirmed in a human setting, our findings may be important for future clinical developments in the field of diagnostics for bladder cancer. Implementing the cost-effective protocol of EB instillations in combination with WL cystoscopy may offer a benefit to patients and the healthcare system compared with the costly photodynamic procedure.

\section{Acknowledgements}

The present study was funded by the KU Leuven (OT project no. OT/11/075). Library preparation, sequencing and statistical analysis were performed by VIB Nucleomics Core (www.nucleomics.be).

\section{References}

1. Ferlay J, Soerjomataram I, Dikshit R, Eser S, Mathers C, Rebelo M, Parkin DM, Forman D and Bray F: Cancer incidence and mortality worldwide: Sources, methods and major patterns in GLOBOCAN 2012. Int J Cancer 136: E359-E386, 2015.

2. Park JC, Citrin DE, Agarwal PK and Apolo AB: Multimodal management of muscle-invasive bladder cancer. Curr Probl Cancer 38: 80-108, 2014.

3. van Rhijn BW, Burger M, Lotan Y, Solsona E, Stief CG, Sylvester RJ, Witjes JA and Zlotta AR: Recurrence and progression of disease in non-muscle-invasive bladder cancer: From epidemiology to treatment strategy. Eur Urol 56: 430-442, 2009.

4. Jocham D, Stepp H and Waidelich R: Photodynamic diagnosis in urology: State-of-the-art. Eur Urol 53: 1138-1148, 2008.

5. Yang LP: Hexaminolevulinate blue light cystoscopy: A review of its use in the diagnosis of bladder cancer. Mol Diagn Ther 18: 105-116, 2014.

6. Cordeiro ER, Anastasiadis A, Bus MT, Alivizatos G, de la Rosette JJ and de Reijke TM: Is photodynamic diagnosis ready for introduction in urological clinical practice? Expert Rev Anticancer Ther 13: 669-680, 2013.

7. Burger M, Grossman HB, Droller M, Schmidbauer J, Hermann G, Drăgoescu O, Ray E, Fradet Y, Karl A, Burgués JP, et al: Photodynamic diagnosis of non-muscle-invasive bladder cancer with hexaminolevulinate cystoscopy: A meta-analysis of detection and recurrence based on raw data. Eur Urol 64: 846-854, 2013.

8. Babjuk M, Böhle A, Burger M, Capoun O, Cohen D, Compérat EM, Hernández V, Kaasinen E, Palou J, et al: EAU Guidelines on Non-Muscle-invasive Urothelial Carcinoma of the Bladder: Update 2016. Eur Urol: Jun 17, 2016 (Epub ahead of print).

9. Roelants M, Huygens A, Crnolatac I, Van Cleynenbreugel B, Lerut E, Van Poppel H and de Witte PA: Evans blue as a selective dye marker for white-light diagnosis of non-muscle-invasive bladder cancer: An in vitro study. BJU Int 109: 300-305, 2012.

10. Elsen S, Lerut E, Van Cleynenbreugel B, Van der Aa F, Van Poppel $\mathrm{H}$ and de Witte PA: Biodistribution of Evans blue in an orthotopic AY-27 rat bladder urothelial cell carincinoma model: Implication for the improved diagnosis of non-muscle-invasive bladder cancer (NMIBC) using dye-guided white-light cystoscopy. BJU Int 116: 468-477, 2015.

11. Gardner MJ: Micromethod for the analysis of evans blue in plasma using ion-pair high-performance liquid chromatography. J Chromatogr 381: 295-303, 1986.

12. Liao Y, Smyth GK and Shi W: FeatureCounts: An efficient general purpose program for assigning sequence reads to genomic features. Bioinformatics 30: 923-930, 2014.

13. Risso D, Schwartz K, Sherlock G and Dudoit S: GC-content normalization for RNA-seq data. BMC Bioinformatics 12: 480, 2011.

14. Robinson MD and Smyth GK: Moderated statistical tests for assessing differences in tag abundance. Bioinformatics 23: 2881-2887, 2007.

15. Benjamini Y and Hochberg Y: Controlling the false discovery rate: A practical and powerful approach to multiple testing. J R Statist Soc B 57: 289-300, 1995.

16. Gibson JG and Evans WA: Clinical studies of the blood volume. I. Clinical application of a method employing the azo dye 'Evans blue' and the spectrophotometer. J Clin Invest 16: 301-316, 1937.

17. Crooke AC and Morris CJ: The determination of plasma volume by the Evans blue method. J Physiol 101: 217-223, 1942.

18. Brown MA, Mitar DA and Whitworth JA: Measurement of plasma volume in pregnancy. Clin Sci (Lond) 83: 29-34, 1992. 
19. Farquhar WB, Hunt BE, Taylor JA, Darling SE and Freeman R: Blood volume and its relation to peak $\mathrm{O}(2)$ consumption and physical activity in patients with chronic fatigue. Am J Physiol Heart Circ Physiol 282: H66-H71, 2002.

20. Boron WF and Boulpaep EL (eds): Arteries and veins. In: Medical physiology. Elsevier Inc., Philadelphia, PA, pp447-462, 2005.

21. Gibson JG and Gregersen MI: Toxicity of two vital dyes used in plasma volume determinations. Am J Physiol (Proc) 113: 50, 1935.

22. Belafsy PC, Blumenfeld L, LePage A and Nahrstedt K: The accuracy of the modified Evan's blue dye test in predicting aspiration. Laryngoscope 113: 1969-1972, 2003.

23. Kanai M, Sakurai T, Yoshinaga K, Aoyagi K, Hitsumoto T, Yoshinuma M, Uchi T, Noike H, Ohsawa H, Kawamura K, et al: Percutaneous dye image cardioscopy for detection of endocardial lesions. Diagn Ther Endosc 7: 29-33, 2000.
24. Hewitt KJ, Agarwal R and Morin PJ: The claudin gene family: Expression in normal and neoplastic tissues. BMC Cancer 6: 186, 2006.

25. Jeanes A, Gottardi CJ and Yap AS: Cadherins and cancer: How does cadherin dysfunction promote tumor progression? Oncogene 27: 6920-6929, 2008.

26. Bryan RT and Tselepis C: Cadherin switching and bladder cancer. J Urol 184: 423-431, 2010.

27. Bryan RT, Atherfold PA, Yeo Y, Jones LI, Harrison FR, Wallace DM and Jankowski JA: Cadherin switching dictates the biology of transitional cell carcinoma of the bladder: Ex vivo and in vitro studies. J Pathol 215: 184-194, 2008. 